Fernandez, Anthony V. (2019). "Phenomenological Psychopathology and Psychiatric Classification." In The Oxford Handbook of Phenomenological Psychopathology, edited by Giovanni Stanghellini, Matthew Broome, Anthony Vincent Fernandez, Paolo Fusar-Poli, Andrea Raballo, and René Rosfort. Oxford University Press.

Please quote from the published version, which is available at the following address:

http://www.oxfordhandbooks.com/view/10.1093/oxfordhb/9780198803157.001.0001/oxfordhb$\underline{9780198803157}$

\title{
Phenomenological Psychopathology and Psychiatric Classification
}

\author{
Anthony Vincent Fernandez, Kent State University
}

\begin{abstract}
In this chapter, I provide an overview of phenomenological approaches to psychiatric classification. My aim is to encourage and facilitate philosophical debate over the best ways to classify psychiatric disorders. First, I articulate phenomenological critiques of the dominant approach to classification and diagnosis-i.e., the operational approach employed in the Diagnostic and Statistical Manual of Mental Disorders (DSM-5) and the International Classification of Diseases (ICD-10). Second, I describe the type or typification approach to psychiatric classification, which I distinguish into three different versions: ideal types, essential types, and prototypes. I argue that despite their occasional conflation in the contemporary literature, there are important distinctions among these approaches. Third, I outline a new phenomenological-dimensional approach. I show how this approach, which starts from basic dimensions of human existence, allows us to investigate the full range of psychopathological conditions without accepting the validity of current diagnostic categories.
\end{abstract}

\section{Keywords}

Classification; Diagnosis; Phenomenology; Types; Typification; Dimensions; DSM; ICD 


\section{Phenomenological Psychopathology and Psychiatric Classification Anthony Vincent Fernandez, Kent State University}

\section{Introduction}

Today, the dominant systems of psychiatric classification are the $5^{\text {th }}$ edition of the Diagnostic and Statistical Manual of Mental Disorders (DSM-5; APA, 2013) and the $10^{\text {th }}$ edition of the International Classification of Diseases (ICD-10; WHO, 2004)—which, in its forthcoming $11^{\text {th }}$ edition, will largely converge with the DSM-5. Other systems include the Psychodynamic Diagnostic Manual (PDM-2; Lingiardi \& McWilliams, 2017), which is informed by psychoanalytic approaches, and the Research Domain Criteria (RDoC) initiative, a dimensional approach developed by the US National Institute of Mental Health (Cuthbert, 2014; Cuthbert \& Insel, 2013). The goal of any psychiatric classification should be to provide a means of organizing and navigating the complex domain of mental disorders, thereby guiding both clinical practice and scientific research.

Most contemporary approaches aim for a biological classification. They assume that mental disorders are, at their basis, biological disease entities. The goal of classification is, therefore, to provide a set of categories that maps onto discrete biological diseases. However, in light of the DSM's failure to biologically validate its categories of disorder, psychiatrists are now looking to alternative approaches that might produce new classifications.

Some of these alternative approaches are grounded in phenomenology, a philosophical study of human experience and existence. Phenomenological psychopathologists describe not only "what it's like" or "what it feels like" to live with a mental disorder, but also how the structural features of human experience and existence-e.g., intentionality, selfhood, temporality, and affectivity — alter in psychopathological cases. At this point, however, none of 
these classificatory approaches have culminated in a classification scheme like the ones we find in the DSM, ICD, or PDM. Here, I refer to a classificatory approach as a process or method of classifying phenomena. In the case of mental disorders, classificatory approaches might track, for instance, differences in experience, behavior, neurobiology, or treatment response. I refer to a classification scheme as the set of phenomena that an approach has grouped or delineated. In the case of the DSM, for instance, the scheme is the set of categories included in the manual.

Therefore, to say that phenomenologists have developed only classificatory approaches means that they have methods for delineating the range of psychopathological conditions, but they have not actually produced their own scheme.

In addressing the topic of phenomenology and psychiatric classification, this chapter has two aims: First, it presents phenomenological critiques of the DSM/ICD approach to classification. Second, it delineates the various phenomenological approaches to classification. My intention is not to take a stand on the adequacy of any particular approach. Rather, my intention is to delineate the central features of these approaches side-by-side, and against the backdrop of the DSM/ICD, so that phenomenologists will be in a better position to debate these approaches and develop more effective systems of classification-e.g., systems that more accurately align with neurobiological markers, predict course of illness, or guide targeted therapeutic interventions.

\section{Phenomenological Critiques of Operationalism}

Since the DSM-III (APA, 1980), operationalism has been the dominant approach to classification and diagnosis. As employed in the DSM, an "operational approach" is one that categorizes and diagnoses disorders by reference to a set of easily observable symptoms, some number of which 
must be present for a predefined period of time. ${ }^{1}$ Major depressive disorder (MDD), for example, is diagnosed when at least five of nine possible symptoms are present for at least two weeks. One of these symptoms must be either (1) depressed mood or (2) loss of interest or pleasure. The other symptoms include (3) significant weight loss or weight gain; (4) insomnia or hypersomnia; (5) psychomotor agitation or retardation; (6) fatigue or loss of energy; (7) feelings of worthlessness or excessive or inappropriate guilt; (8) diminished ability to think or concentrate; and (9) recurrent thoughts of death or suicidal ideation (APA, 2013). Schizophrenia, by contrast, is diagnosed when at least two of five symptoms are present over a one-month period: (1) delusions; (2) hallucinations; (3) disorganized speech; (4) grossly disorganized or catatonic behavior; and (5) negative symptoms; (in addition, at least one of the symptoms must be items 1, 2, or 3) (APA, 2013). ${ }^{2}$

Notably, the DSM's major focus has been on enhancing interrater reliability—-that is, increasing the likelihood that two or more clinicians will diagnose the same patient with the same condition. An operational approach, with its reliance on easily observable symptoms and structured diagnostic interviews provides an effective means of enhancing reliability. But psychiatrists want more than reliability - they want validity. In most cases, this takes the form of an appeal to neurobiological underpinnings. However, as Assen Jablensky and Robert Kendell point out, there are numerous definitions of validity in psychiatry, with little agreement on which sense of validity should be privileged. A category might be considered valid if it

\footnotetext{
${ }^{1}$ In contemporary psychiatry, what goes under the label of "operationalism" is relatively superficial when compared with its early philosophical and scientific development in the work of Carl Hempel (1966), Edwin Boring (1945), and Percy Williams Bridgman (1938). While psychiatry's version of operationalism likely has its roots in this history, one should not assume that it takes over the fairly robust philosophical programs of these early figures. For a more detailed account of the history of operationalism in psychology and psychiatry, see Hasok Chang (2009) and Josef Parnas and Pierre Bovet (2015).

${ }^{2}$ Each diagnostic category includes additional criteria, such as significant impairment of one's ability to work, engage in self-care, or maintain social relations.
} 
(a) is based on a coherent, explicit set of defining features (construct validity); (b) has empirical referents, such as verifiable observations for establishing its presence (content validity); (c) can be corroborated by independent procedures such as biological or psychological tests (concurrent validity); and (d) predicts future course of illness or treatment response (predictive validity). (Jablensky \& Kendell, 2002, p. 10)

In light of this state of affairs, some psychiatrists—including Jablensky and Kendell—argue that we should appeal to a concept of utility instead of validity. Utility is achieved when a category or scheme "provides nontrivial information about prognosis and likely treatment outcomes, and/or testable propositions about biological and social correlates" (Kendell \& Jablensky, 2003, p. 9). Considering the DSM's shortcomings with respect to validity and utility, it has been the subject of widespread criticism from groups both inside and outside of mainstream psychiatry.

Phenomenological psychopathologists constitute one of these critical groups.

Phenomenologists don't necessarily agree on how to approach the problem of validity or utility — some seem to endorse, for example, the view that phenomenology should assist in the process of distinguishing biological disease entities, whereas others defend a less naturalistic view. There are, however, three general criticisms of the DSM's operational approach that we find in the phenomenological literature:

1) It ignores how clinicians intuitively diagnose patients.

2) It neglects the organization among the various symptoms, treating them as disparate parts without a unified whole.

3) It allows categories to reify, inhibiting future refinement and revision of the classification scheme. ${ }^{3}$ (Parnas \& Bovet, 2015; Schwartz \& Wiggins, 1987a, 1987b)

In general, phenomenological approaches to psychiatric classification claim to resolve all of these issues. In what follows, I outline four phenomenological approaches to classification,

${ }^{3}$ This situation is even more problematic when a diagnostic category encompasses a heterogeneity of conditions, as we find in the case of MDD (see, e.g., Ratcliffe, Broome, Smith, \& Bowden, 2013) 
which I divide into two broad classes: type and dimension approaches. The first class includes ideal types, essential types, and prototypes. The second class includes a dimensional approach that I have recently proposed, which draws inspiration from the National Institute of Mental Health's Research Domain Criteria (RDoC) initiative.

\section{Typification and Classification}

Before distinguishing the ideal type, essential type, and prototype approaches, it will be helpful to outline what they hold in common. All of these approaches aim to resolve the above shortcomings of the DSM's operational approach, and they do so in the following ways:

1) They take stock of how clinicians intuitively understand their patients - utilizing, rather than ignoring, this intuitive understanding.

2) They seek an organizing principle, a gestalt, or what Eugene Minkowski calls the trouble générateur, that makes sense of the total structure of the disorder.

3) They build in mechanisms for continued revision and refinement, treating current concepts as one step along the way to a better system of classification.

With respect to the first solution, all three approaches share the basic phenomenological insight that we perceive and make sense of our world through tacit types or categories. In everyday experience, these types are not explicitly defined and their boundaries are not always clear. Whenever we perceive something, we perceive it as some particular kind of thing; we never perceive a bare object. For example, if I walk through my garden and see that a rose has bloomed, I simply see it as a rose. In most cases, I don't list off explicit criteria to check whether it's a rose. I just know what a rose looks like. 
But this doesn't mean that our typifications are never wrong. Perhaps I invite my friend, a botanist, over to show her my rose. Upon walking into the garden, she exclaims, "That's not a rose! It's a peony." Realizing that my initial typification was incorrect, I'm now open to being corrected by my friend. When she corrects me, she might teach me explicit criteria for distinguishing roses from peonies. And I might rely on these explicit criteria for some time. But, eventually, this too will become habitual and tacit. I'll develop my own expertise, immediately seeing a flower as a rose, a peony, or some other species.

Our typifications are, therefore, learned and refined in the course of everyday experience. I wasn't born knowing how to identify flowers; I learned what counted as a flower and how to distinguish among different kinds of flowers through everyday experience (and with a little guidance from my parents). And, even now, my ability to tacitly typify or categorize flowers remains relatively limited. The botanist is not only better at distinguishing peonies from roses; she can also distinguish among a wide variety of peonies and roses. And, as an expert, she can distinguish them intuitively, without appealing to explicit criteria. In this respect, to refer to the process of typification as intuitive simply means that it occurs tacitly or implicitly—not that it's based on an innate or unchanging base of knowledge.

Moreover, seeing as isn't simply a process of identifying and categorizing objects within the world. In addition to identifying and categorizing, I also have an immediate sense of how to take things up and put them to use. For example, when I see an object on my table as a mug, it immediately shows up to me as a vessel for holding tea, coffee, or hot chocolate. It also shows up to me as something I should wash in the kitchen sink or place in the dishwasher. In general, these actions aren't things that I have to explicitly reflect on or infer. To see an object as a mug 
just is to see it as something that can perform these functions and should be handled in these ways.

Phenomenologists argue that we employ tacit typifications not only in our experience of everyday objects, but also in our experience of other people. To navigate the social world, we typify people in a variety of ways and these typifications guide our initial interactions. When I walk into my classroom, I immediately perceive the people before me as my students and they perceive me as their professor. These tacit typifications shape how we engage each other, determining proper modes of address, expected behaviors, and so on. Such typifications can, of course, be pernicious: They might, for instance, become racial or gender stereotypes. However, phenomenologists argue that prejudices aren't inherently problematic (see, e.g., Gadamer, 1960/2013, 2008). We need presuppositions and assumptions in order to successfully navigate our environment (although we also need to be open to revising them in light of future experiences).

But what does everyday typification have to do with the practice of clinical psychology and psychiatry? Proponents of typification argue that clinicians have an intuitive understanding of their patients, and that this understanding often leads to an initial diagnosis (Schwartz \& Wiggins, 1987b). In the clinical encounter, the skilled clinician doesn't simply perceive a generic person who happens to exhibit an array of symptoms. Her perception often includes a tacit sense of her patient as depressed, as schizophrenic, as autistic, and so on. This initial perception can guide her process of questioning, helping her arrive at a final diagnosis. The operational, checklist diagnostic method of the DSM-5 ignores this skilled, intuitive mode of understanding and therefore fails to put it to use. 
Over the past few decades, intuitive diagnosis has been overshadowed by the operational approach. However, there has been a recent renewal in studies of the effectiveness and accuracy of intuitive modes of diagnosis, focusing both on the diagnosis of schizophrenia (Gozé et al., 2018) and on the ability to identity those at risk of developing schizophrenia (Lindau et al., n.d.). These studies stress that the intuitive approach provides only a preliminary or tentative diagnosis. An expert psychiatrist should be guided by his typifications, but should not simply allow his initial intuition to determine the final diagnosis. Marcin Moskalewicz, Michael Schwartz, and Tudi Gozé (2018) argue that intuitive diagnosis, properly conducted, is not simply an immediate sense of the patient's condition. Rather, it is a temporally extended process in which the psychiatrist critically reflects upon his intuition or typification and seeks out disconfirming evidence. Moreover, they claim that this mode of diagnosis need not conflict with an operational approach. One might, for instance, be led to an initial diagnostic category through intuition, but confirm or disconfirm this intuition by employing operational criteria.

This practice of actively confirming or disconfirming an intuitive diagnosis is linked with the practice of revising and refining diagnostic categories themselves. This latter practice is as an attempt to make the diagnostic system more rigorous and scientific. We need to guarantee that our types accurately identify the conditions they're meant to diagnose and that these types don't reify into outdated and inaccurate categories. However, the three typification approaches do not necessarily agree on how we should make diagnosis scientific. Each proposes a slightly different method of harnessing everyday typifications, transforming them into scientific concepts and categories (Fernandez, 2016).

These approaches are not, however, properly disambiguated in the literature. The essential type and prototype approaches, for instance, are sometimes presented as if they don't 
differ in any important respects from the ideal type approach. To head off potential confusions, I describe each approach below by focusing on their distinctive features, illustrating how each proposes a different way of making typifications scientific — and, ultimately, a different way of classifying mental disorders.

\subsection{Ideal Types}

The ideal type approach is championed by Schwartz and Osborne Wiggins. In the fields of psychology and psychiatry, this approach is rooted in Karl Jaspers' General Psychopathology (1913/1997). But Jaspers himself adapted it from Max Weber's initial proposal. Weber, the founder of modern sociology, argued that social phenomena could not be classified in the same manner as natural objects. The sociologist cannot create a classification akin to the periodic table of elements because social phenomena do not have hard boundaries - their manifestations are fluid and variable. To better capture the features of social phenomena, Weber developed the notion of an ideal type. As he defines it,

An ideal type is formed by the one-sided accentuation of one or more points of view and by the synthesis of a great many diffuse, discrete, more or less present and occasionally absent concrete individual phenomena, which are arranged according to those onesidedly emphasized viewpoints into a unified analytical construct (Gedankenbild). In its conceptual purity, this mental construct (Gedankenbild) cannot be found empirically anywhere in reality. It is a utopia. (Weber, 1949, p. 90)

What is most important here - at least with respect to differentiating the phenomenological approaches to classification - is that the ideal type is not an empirical reality. It is ideal in the sense of being pure, uncontaminated by the messy details of the concrete world (Broome, 2006). 
But what makes ideal types scientific? As Schwartz and Wiggins explain, ideal types are heuristic devices. They provide the community of researchers and clinicians with a means of conceptually organizing their subject matter. As heuristic devices, ideal types aren't taken as "true"-i.e., they aren't judged with respect to how accurately they represent some aspect of reality. Rather, by characterizing her concept as an ideal type, the researcher or clinician admits that no instance of the disorder in question will correspond exactly with her concept; the concrete manifestation might lack certain features included in the ideal type, or express additional features not included in the ideal type (Schwartz \& Wiggins, 1987a, p. 283). And, as Schwartz and Wiggins argue, this lack of perfect fit enhances the scientific value of ideal types; the precise ways in which the concrete instance of the disorder fails to correspond with the ideal type can guide the processes of both clinical diagnosis and scientific research.

The clinician's goal—as Schwartz and Wiggins present it—is to understand the patient in his particularity. Therefore, the ways in which the patient diverges from the ideal type can guide further inquiry into the unique nature of his individual condition. One of the researcher's goals, by contrast, is to produce a naturalistic or biological classification. ${ }^{4}$ In this case, the ideal type provides a shared starting point for constructing hypotheses and carrying out experiments. As Schwartz and Wiggins explain,

For psychiatric research, ideal types furnish the initial conceptual guidelines for the postulation of law-like regularities and the design of experiments to test such postulates. For clinical practice, ideal types predelineate the features of disorders so that clinicians

\footnotetext{
${ }^{4}$ Among phenomenologists, it's by no means universally accepted that a biological classification should be our ultimate goal. However, in at least some of Schwartz and Wiggins' presentations of their program, the ideal type approach is characterized as a step along the way to a biological classification.
} 
know what to search for, focus on, and examine in particular patients. (Schwartz \& Wiggins, 1987a, p. 286)

Because ideal types are heuristic devices, their value is based entirely on their utility. If the community of researchers and clinicians finds that altering the ideal type (or replacing it altogether) better guides their research and clinical practice, then they are free to make such alterations or replacements. In this respect, the set of ideal types used to delineate the field of mental disorders is not taken as a definitive system of classification. It is one step along the way. ${ }^{5}$

\subsection{Essential Types}

The essential type approach was proposed by Josef Parnas and Dan Zahavi (2002) (although they do not call it by this name). Parnas and Zahavi ground their approach in Husserlian phenomenology: "a tradition specifically aiming at grasping the essential structures of human experience and existence" (2002, p. 143). However, there is an ambiguity in their adaptation of this approach to the study of mental disorders. They initially characterize their phenomenological approach to psychiatric diagnosis and classification as if it is identical with Schwartz and Wiggins' approach:

A concept of "ideal type" or "essence" plays here an important role. Ideal type exemplifies the ideal and necessary connections between its composing features. Ideal type transcends what is given in experience: e.g. all my possible drawings of a straight line will be somehow deficient (for instance if examined through a microscope) compared to the very (ideal) concept of a straight line. (Parnas \& Zahavi, 2002, p. 157).

\footnotetext{
${ }^{5}$ This characterization is, notably, in contrast with Weber's initial employment of ideal types. Weber's goal was not to use ideal types as a step toward a naturalistic conceptualization of social phenomena. Rather, he thought that social phenomena were best classified through ideal types.
} 
Here, Parnas and Zahavi appeal directly to Schwartz and Wiggins' (1987a) ideal types. But their further characterizations seem to present a substantially different project; as Matthew Broome has pointed out, Parnas and Zahavi seem to conflate Weberian ideal types with Husserlian essences (Broome, 2006, p. 311).

If we look further into how Parnas and Zahavi characterize their approach, we find a key distinction between ideal types and essential types, which hinges on their relation to necessity. According to Parnas and Zahavi, the "[p]henomenological approach to anomalous experience is precisely concerned with bringing forth the typical, and ideally necessary features of such experience" (2002, p. 157; my emphasis). As they explain, their approach identifies essential features of categories of mental disorder, rather than just essential features of human experience as such. In order to discover these essential features, they suggest that that we employ Husserl's eidetic reduction, which involves a process of imaginative variation:

This process of imaginative variation will lead us to certain borders that cannot be varied, i.e. changed and transgressed, without making the phenomenon cease to be the kind of phenomenon it is. The variation consequently allows us to distinguish between the accidental properties, i.e. the properties that could have been different, and the essential properties, i.e. the invariant structures that make the phenomenon be of the type it is. (Parnas \& Zahavi, 2002, p. 157)

On this approach, a condition that fails to display an essential feature simply won't count as the disorder in question. Parnas and Zahavi's emphasis on essential features will, therefore, produce more rigid categories than Schwartz and Wiggins' ideal types because ideal types do not identify necessary features of disorders; they are heuristic devices with no truth value. But this is not to suggest that Parnas and Zahavi's categories will not be amenable to revision and refinement. As 
they explain, to claim that something is an essential feature of a disorder is not to claim that this has been proved once and for all. It's possible that one was mistaken in labeling a feature "essential" Future phenomenological analyses might provide reason to believe that the feature is contingent or accidental (even if common or typical) (Parnas \& Zahavi, 2002, p. 157).

\subsection{Prototypes}

The prototype approach, like ideal type and essential type approaches, is intended to make the everyday process of typification scientific. Parnas and Shaun Gallagher (2015) propose prototypes as an alternative to operational classification and diagnosis. According to Parnas and Gallagher, "A prototype is a central example of a category in question (a sparrow is more characteristic of the category "bird" than is a penguin or an ostrich), with a graded dilution of typicality toward the borders of the category, where it eventually overlaps exemplars from neighboring categories" (2015, p. 73; my emphasis). As they characterize it here, a prototype is neither a utopic ideal nor a clearly defined essence. Unlike an ideal type, a prototype is a concrete exemplar of the disorder, which necessarily implies that such an exemplar actually exists. And, unlike an essential type, a prototype does not establish a set of necessary and essential features of the condition in question. Rather, the prototype is itself a concrete instance of the condition. Other conditions are categorized based on how closely they match this particular instance.

Moreover, Parnas and Gallagher characterize their particular version of prototypes as a "prototype-gestalt," which highlights the unity and organization of symptoms. However, an apparent contradiction arises in their characterization of the gestalt. They say, "A gestalt instantiates a certain generality of type. Yet, this type-generality is always deformed, because it 
inheres in a particular, concrete and situated individual. The particular token always attenuates the ideal clarity and pregnancy of type" $(2015$, p. 75$)$. By suggesting that there is no perfect, concrete instance of the condition, Parnas and Gallagher characterize their approach as if it's akin to Schwartz and Wiggins' ideal types - and they refer directly to Schwartz and Wiggins' work on the topic (1987a, 1987b). But this seems to contradict their initial characterization: They initially argued that—on their prototype approach—categories are defined, or anchored, by appealing to a concrete exemplar. But if the prototype or exemplar is an imperfect instantiation of its category — as they suggest in their second characterization — then it can't be what's anchoring the category; the prototype does not stand, as it were, in the center of the category. Parnas and Gallagher seem to appeal to an ideal category with respect to which the prototype itself is judged as adequate or inadequate.

I don't mean to suggest that this apparent contradiction is necessarily unresolvable. There might be some manner of reconciling these two approaches: For example, the prototype might be a useful diagnostic or teaching tool, while the ideal type is what the psychiatrist ultimately appeals to when revising his classificatory scheme. However, Parnas and Gallagher don't seem to acknowledge the apparent contradiction in the first place, and therefore offer no resolution of their own. ${ }^{6}$

\footnotetext{
${ }^{6}$ These ambiguities are compounded by Parnas and Gallagher's apparent use of "prototype" and "exemplar" as synonyms. In their initial characterization of their prototype approach, they appeal to Edouard Machery's work on concept formation. But Machery clearly distinguishes between "prototype" and "exemplar" approaches to concept formation. As he describes it, "a prototype of a class is a body of statistical knowledge about the properties deemed to be possessed by members of this class" (Machery, 2009, p. 83). An exemplar, by contrast, "is a body of knowledge about the properties believed to be possessed by a particular member of a class" (Machery, 2009, p. 93). In light of this, it seems that Parnas and Gallagher's proposal is akin to an "exemplar" approach, which therefore diverges substantially from what's often called a "prototype" approach.
} 
In addition to this ambiguity, another confusion arises when we consider Schwartz, Wiggins, and Michael Norko's $(1989,1995)$ early work on the relationship between ideal types and prototypes. However, I believe this confusion can be avoided once we understand that their critique of prototypes does not apply to the version presented by Parnas and Gallagher. Schwartz, Wiggins, and Norko suggest that prototypes have two major shortcomings: First, prototypes consist of a set of attributes without conceptual unity; Jaspers, for example, says that a prototype is "a disjointed enumeration" of features (Schwartz et al., 1989, p. 6). Second, prototypes cannot produce novel concepts because they are constructed by surveying psychiatrists about how they diagnose and understand their patients - prototypes merely reflect the current understanding of mental disorders (1989, p. 6). However, from the above account, it should be clear that neither of these critiques apply to Parnas and Gallagher's prototype approach. Despite the ambiguities in their approach, they clearly posit a gestalt organization among the prototype's features and they do not believe that prototypes are constructed by merely surveying and averaging typical ways of understanding mental disorders.

\section{Dimensions}

Until recently, all phenomenological approaches to classification and diagnosis have been type approaches. However, I have proposed a phenomenological-dimensional approach to psychiatric research and classification (Fernandez, forthcoming) ${ }^{7}$ The primary motivation behind this approach is psychiatry's recent shift from categorial approaches to dimensional approaches. By “dimensional," I do not refer to the DSM-5's construction of new, graded categories, such as autism spectrum disorder. Rather, I refer to approaches that dispense with categories altogether,

${ }^{7}$ Thomas Fuchs and Mauro Pallagrosi have also proposed the use of a dimensional approach for the study of psychopathological temporality (Fuchs \& Pallagrosi, 2018). 
beginning, instead, from basic aspects of human experience or behavior. If psychiatry continues in this direction—which seems likely—then the gulf between phenomenology's categorial approach and mainstream psychiatry will only widen. In light of this, I've suggested that phenomenologists embrace a broadly dimensional approach, thereby reducing barriers to interdisciplinary collaboration between phenomenologists and psychiatrists.

Psychiatry's major dimensional approach is the Research Domain Criteria (RDoC) initiative developed by the US National Institute of Mental Health. The RDoC aims to produce a new research classification (i.e., one not currently intended for use in clinical practice) based on dimensions or constructs (the terms are used interchangeably) of human experience and behavior. Constructs are clustered into broad domains, such as Cognitive Systems or Social Processes. The Social Processes domain includes, for example, the constructs of Affiliation and Attachment, Social Communication, Perception and Understanding of Self, and Perception and Understanding of Others. Some of these constructs are then divided into subconstructs. For example, Perception and Understanding of Self includes the subconstructs of Agency and SelfKnowledge. By orienting research through this matrix, researchers achieve two ends: First, they can investigate psychopathological conditions without framing their investigations through the lens of invalid DSM categories. Second, they can study how this dimension manifests across both normal and abnormal conditions; they might study, for instance, how agency manifests across subjects who would typically be diagnosed with depression, bipolar disorder, and schizophrenia.

How can we develop a dimensional approach for use in phenomenology? While a broadly dimensional approach might draw inspiration from the RDoC, it need not conform with the RDoC matrix (i.e., its set of domains, constructs, and subconstructs). I have argued that a 
phenomenological matrix of domains and dimensions can be drawn from what Heidegger calls "existentials," and other phenomenologists refer to as "transcendental," "essential," or “ontological" structures (Fernandez, 2017, forthcoming). Each existential is a basic feature of human existence, such as intentionality, selfhood, affectivity, or temporality. These existentials constitute the basic domains of phenomenological psychopathology, with each existential further distinguished into its various structural moments, or features, which constitute its dimensions. The domain (or existential) of Selfhood, for instance, can include the dimensions of Core Self and Narrative Self, with each dimension further subdivided or specified as required. The Core Self might include features such as (1) Cognition and Stream of Consciousness; (2) SelfAwareness and Presence; (3) Bodily Experience; and (4) Demarcation/Transitivism of the selfworld boundary (see Parnas et al., 2005) ${ }^{8}$ We can then investigate each of these domains, constructs, and subconstructs across the full range of normal and abnormal experience, moving beyond current diagnostic categories. ${ }^{9}$

My phenomenological-dimensional approach is —at this time — only a proposal. However, some phenomenologists have already conducted studies with a broadly dimensional outlook. I briefly outline two of these studies here with the intention of providing some insight into what a phenomenological-dimensional approach might look like in practice. First, I outline Louis Sass and Elizabeth Pienkos' comparative study of selfhood across melancholia, bipolar mania, and schizophrenia. Second, I outline Matthew Ratcliffe's study of temporal disturbances in depressive disorders. While these authors don't explicitly characterize their work as

\footnotetext{
${ }^{8}$ These items are adapted from the Examination of Anomalous Self Experience (EASE) (Parnas et al., 2005). As I've argued elsewhere, the EASE does not translate directly into the kind of dimensional approach I am proposing, but some of its items can be appropriated for dimensional analysis (Fernandez, forthcoming).

${ }^{9}$ For a more complete account of a phenomenological-dimensional approach, including illustrative examples, see Fernandez (forthcoming).
} 
dimensional in the sense I've described here, these studies investigate how basic existential structures alter across psychopathological conditions; they're framed through what I've called the basic domains and dimensions of human existence, rather than through current diagnostic categories.

Sass and Pienkos study how one dimension of experience — the core self—alters across three categories of disorder. One feature of the core self that they explore is the experience of the self-world boundary, or the way in which I experience myself and my identity in relation with my environment. As they argue, in melancholia the boundary between self and world is enhanced or increased; the world feels distant or unreachable. In mania, by contrast, the boundary between self and world is significantly diminished, sometimes culminating in an experience of mystic union (Sass \& Pienkos, 2013, p. 124). We might assume that this manic experience is akin to schizophrenia, which also involves a diminished self-world boundary. However, Sass and Pienkos argue that this apparent similarity is superficial. If we carefully attend to the way this dimension of experience can change, we'll find that there are important differences in the experience of the self-world boundary between mania and schizophrenia. The experiences differ in two key respects: First, in mania the diminished self-world boundary is typically accompanied by a positive or neutral mood, whereas in schizophrenia it is typically accompanied by feelings of anxiety in which the subject feels invaded by the external world. Second, in mania there is often a sense of oneness with the world, whereas schizophrenia can include a sense of solipsism (the world is produced by my mind and has no independent reality).

While Sass and Pienkos' study still refers to current diagnostic categories, the categories don't frame their investigation. Rather, their investigation is framed through the dimension of selfhood, which allows them to test and further articulate categorial boundaries. Such an 
investigation would be impossible if a study were confined to a particular category or had to assume the validity of current diagnostic categories from the start. Moreover, this suggests that dimensional and categorial approaches need not be mutually exclusive. A dimensional approach might help us refine clinical diagnostic categories.

Another broadly dimensional approach is employed in Matthew Ratcliffe's study of temporal disturbances in depressive disorders. Instead of comparing different diagnostic categories, Ratcliffe uses a dimensional analysis to assess the alleged homogeneity of depressive disorders. By showing that people diagnosed with depression report a variety of temporal disturbances, he casts doubt on the belief that we've identified a single, unified condition, and motivates the project of reclassifying depressive disorders.

Many people with depression report changes in their temporal experience, such as the sense of time slowing down or stretching out (similar to the temporal experience of boredom). Ratcliffe, however, argues that if we attend more carefully to descriptions of temporal experience in depressive episodes, we'll find a wide variety of temporal disturbances expressed across these reports. Ratcliffe clusters these disturbances into three groups: loss of significane, loss of conative drive, and loss of life projects (Ratcliffe, 2012). First, some people report that future possibilities are not enticing or worth pursuing. Among this group, some report that future possibilities are insignificant as such—i.e., future possibilities are experienced as having no significance for anyone. Others report that possibilities are insignificant for themselves but understand that others might experience them as significant. Second, some people report that future possibilities are significant and worth pursuing, yet they lack any drive or motivation to pursue them. Third, some people report that future possibilities don't show up at all; they have no sense of who they might be in the future. This is often accompanied by a sense that one will 
never escape their depression (Ratcliffe, 2012, p. 121; see also Aho, 2013; Fernandez, 2014; Maiese, 2017). Any of these disturbances might be experienced independently of the others, but there are also cases in which multiple disturbances occur simultaneously.

Ratcliffe concludes that the diversity of temporal disturbances across people diagnosed with depressive disorders — specifically, with major depressive disorder — suggests that we might not be dealing with one homogeneous condition. There might be a heterogeneity of conditions that, due to imprecise diagnostic criteria, are classed as a single category of disorder. As Ratcliffe says,

I have suggested that "depression" and more specific subcategories of depression such as "major depression" encompass a range of subtly different changes in the structure of temporal experience. I have not attempted to provide a comprehensive taxonomy here. However, I have offered the beginnings of an interpretive framework for doing so and explored at least some of the variety. (Ratcliffe, 2012, p. 134)

While Ratcliffe doesn't provide a new classification scheme of depressive disorders, he does provide some tools that might help us construct a new scheme. These examples suggest that a dimensional approach can undermine the validity of current diagnostic categories, motivate the need for a new classification, and provide new conceptual distinctions that can be used to reclassify psychopathological conditions.

While I believe there are good reasons for phenomenologists to move toward a dimensional approach, I want to suggest that this new approach might be compatible with type approaches. Type approaches seek the basic organizing principle of the disorder in question - the trouble générateur or core gestalt (Parnas, 2012). They aim not only to understand the relationship between parts and whole, but also to identify the core disturbance from which the 
various symptoms arise. In the case of schizophrenia, for instance, many phenomenologists have argued that the variety of symptoms can be tied to a core disturbance in the structure of selfhood; once we understand how selfhood has altered in schizophrenia, we can make sense of a variety if experiences, such as hallucinations as well as delusions of thought insertion and alien control. These experiences arise because of a breakdown in the sense of agency and ownership of one's thoughts, feelings, and perceptions. A dimensional approach, with its focus on identifying and articulating alterations in specific domains and dimensions of human existence, provides a method of identifying these core disturbances. If such an approach is viable, then phenomenology's type and dimension approaches will not only coexist, but can be mutually complementary (Fernandez, forthcoming).

\section{Conclusion}

In this chapter I have provided an overview of phenomenological approaches to psychiatric classification. First, I articulated phenomenological critiques of the operational approach: (1) It ignores how clinicians intuitively diagnose patients, (2) it neglects the organization among the various symptoms, and (3) it allows categories to reify. Second, I described three different type or typification approaches to psychiatric classification-ideal types, essential types, and prototypes - and showed how these approaches differ (despite their occasional conflation in the contemporary literature). Third, I outlined a phenomenological-dimensional approach to psychiatric classification, which starts from existentials, or basic domains of human existence, rather than current diagnostic categories.

My intention, however, was not to argue for the superiority of the dimensional approach over type approaches. Rather, the primary aim of this chapter is to encourage and facilitate 
philosophical debate over the best ways to classify psychiatric disorders. Phenomenology has the potential to make substantial contributions to the project of psychiatric classification. But it's internal ambiguities and inconsistences will need to be resolved before phenomenologists can move forward with a classification scheme of their own. This overview of the field should provide a useful starting point for future dialogue and debate. 


\section{References}

Aho, K. (2013). Depression and embodiment: phenomenological reflections on motility, affectivity, and transcendence. Medicine, Health Care and Philosophy, 16(4), 751-759.

American Psychiatric Association. (1980). Diagnostic and Statistical Manual of Mental Disorders, 3rd Edition. Washington, D.C.: The American Psychiatric Association.

American Psychiatric Association. (2013). Diagnostic and statistical manual of mental disorders, 5th edition: DSM-5. Washington, D.C.: American Psychiatric Publishing.

Boring, E. G. (1945). The use of operational definitions in science. Psychological Review, 52(5), 243-245.

Bridgman, P. W. (1938). Operational analysis. Philosophy of Science, 5(2), 114-131.

Broome, M. (2006). Taxonomy and Ontology in Psychiatry: A Survey of Recent Literature. Philosophy, Psychiatry, \&amp; Psychology, 13(4), 303-319.

Chang, H. (2009). Operationalism. In E. N. Zalta (Ed.), The Stanford Encyclopedia of Philosophy (Fall 2009). Metaphysics Research Lab, Stanford University. Retrieved from https://plato.stanford.edu/archives/fall2009/entries/operationalism/

Cuthbert, B. N. (2014). The RDoC framework: facilitating transition from ICD/DSM to dimensional approaches that integrate neuroscience and psychopathology. World Psychiatry, 13(1), 28-35.

Cuthbert, B. N., \& Insel, T. R. (2013). Toward the future of psychiatric diagnosis: The seven pillars of RDoC. BMC Medicine, 11(1), 126.

Fernandez, A. V. (forthcoming). Phenomenology and Dimensional Approaches to Psychiatric Research and Classification. Philosophy, Psychiatry, \& Psychology. 
Fernandez, A. V. (2014). Depression as Existential Feeling or De-Situatedness? Distinguishing Structure from Mode in Psychopathology. Phenomenology and the Cognitive Sciences, 13(4), 595-612.

Fernandez, A. V. (2016). Phenomenology, Typification, and Ideal Types in Psychiatric Diagnosis and Classification. In R. Bluhm (Ed.), Knowing and Acting in Medicine (pp. 39-58). Lanham, MD: Rowman and Littlefield International.

Fernandez, A. V. (2017). The Subject Matter of Phenomenological Research: Existentials, Modes, and Prejudices. Synthese, 194(9), 3543-3562.

Fuchs, T., \& Pallagrosi, M. (2018). Phenomenology of Temporality and Dimensional Psychopathology. In M. Biondi, M. Pasquini, A. Picardi (Eds.), Dimensional Psychopathology (pp. 287-300). Springer International Publishing.

Gadamer, H.-G. (2008). Philosophical Hermeneutics. (D. E. Linge, Ed.). Berkeley: University of California Press.

Gadamer, H.-G. (2013). Truth and Method (Revised 2nd Edition). London; New York: Bloomsbury Academic. (Original work published 1960)

Gozé, T., Moskalewicz, M., Schwartz, M. A., Naudin, J., Micoulaud-Franchi, J.-A., \& Cermolacce, M. (2018). Is "praecox feeling” a phenomenological fossil? A preliminary study on diagnostic decision making in schizophrenia. Schizophrenia Research. https://doi.org/10.1016/j.schres.2018.07.041

Hempel, C. (1966). Philosophy of Natural Science. Upper Saddle River, NJ: Prentice Hall. Jablensky, A., \& Kendell, R. E. (2002). Criteria for assessing a classification in psychiatry. In M. Maj, W. Gaebel, J. J. López-Ibor, \& N. Sartorius (Eds.), Psychiatric diagnosis and classification. New York: John Wiley \& Sons. 
Jaspers, K. (1997). General psychopathology. (J. Hoenig \& M. W. Hamilton, Trans.). Baltimore, MD: Johns Hopkins University Press. (Original work published 1913)

Kendell, R., \& Jablensky, A. (2003). Distinguishing between the validity and utility of psychiatric diagnoses. American Journal of Psychiatry, 160(1), 4-12.

Lindau, J. F., Broome, M., Cipriani, A., Brandizzi, M., Masillo, A., Catone, G., ... Nastro, P. F. (Unpublished Manuscript). Clinical Impression Assessment of the Pre-psychotic Phase of Schizophrenia (CIAPPS): a screening questionnaire.

Lingiardi, V., \& McWilliams, N. (Eds.). (2017). Psychodynamic Diagnostic Manual, Second Edition: PDM-2. New York: The Guilford Press.

Machery, E. (2009). Doing without Concepts. Oxford: Oxford University Press.

Maiese, M. (2018). Getting stuck: Temporal desituatedness in depression. Phenomenology and the Cognitive Sciences, 17(4), 701-718.

Moskalewicz, M., Schwartz, M. A., \& Gozé, T. (2018). Phenomenology of Intuitive Judgment: Praecox-Feeling in the Diagnosis of Schizophrenia. AVANT. Trends in Interdisciplinary Studies, $9(2)$.

Parnas, J. (2012). The core gestalt of schizophrenia. World Psychiatry, 11(2), 67-69.

Parnas, J., \& Bovet, P. (2015). Psychiatry made easy: Operation(al)ism and some of its consequences. In K. Kendler \& J. Parnas (Eds.), Philosophical issues in psychiatry III: The nature and sources of historical change (pp. 190-212). Oxford: Oxford University Press.

Parnas, J., \& Gallagher, S. (2015). Phenomenology and the interpretation of psychopathological experience. In L. J. Kirmayer, R. Lemelson, \& C. A. Cummings (Eds.), Re-visioning 
psychiatry: Cultural phenomenology, critical neuroscience, and global mental health (pp. 65-80). New York: Cambridge University Press.

Parnas, J., Møller, P., Kircher, T., Thalbitzer, J., Jansson, L., Handest, P., \& Zahavi, D. (2005). EASE: Examination of anomalous self-experience. Psychopathology, 38(5), 236-258.

Parnas, J., \& Zahavi, D. (2002). The role of phenomenology in psychiatric diagnosis and classification. In M. Maj, W. Gaebel, J. J. López-Ibor, \& N. Sartorius (Eds.), Psychiatric diagnosis and classification (pp. 137-162). New York: John Wiley \& Sons.

Ratcliffe, M. (2012). Varieties of temporal experience in depression. Journal of Medicine and Philosophy, 37(2), 114-138.

Ratcliffe, M., Broome, M., Smith, B., \& Bowden, H. (2013). A bad case of the flu? The comparative phenomenology of depression and somatic illness. Journal of Consciousness Studies, 20(7-8), 198-218.

Sass, L., \& Pienkos, E. (2013). Varieties of self-experience: A comparative phenomenology of melancholia, mania, and schizophrenia, Part I. Journal of Consciousness Studies, 20(78), 103-130.

Schwartz, M. A., \& Wiggins, O. P. (1987a). Diagnosis and Ideal Types: A Contribution to Psychiatric Classification. Comprehensive Psychiatry, 28(4), 277-291.

Schwartz, M. A., \& Wiggins, O. P. (1987b). Typifications: The first step for clinical diagnosis in psychiatry. The Journal of Nervous and Mental Disease, 175(2), 65-77.

Schwartz, M. A., Wiggins, O. P., \& Norko, M. A. (1989). Prototypes, ideal types, and personality disorders: The return to classical psychiatry. Journal of Personality Disorders, 3(1), 1-9. 
Schwartz, M. A., Wiggins, O. P., \& Norko, M. A. (1995). Prototypes, ideal types, and personality disorders: The return to classical phenomenology. In W. J. Livesley (Ed.), The DSM-IV personality disorders (pp. 417-432). New York: Guilford Press.

Weber, M. (1949). Methodology of social sciences. (H. A. Finch \& E. A. Shils, Eds.). Glencoe, IL: The Free Press.

World Health Organization. (2004). International Statistical Classification of Diseases and Related Health Problems: 10th Revision. World Health Organization. 